\title{
BRST SUPERGROUPS AND QUANTIZATION
}

\author{
V ALDAYA ${ }^{\text {a }}$, R LOLL ${ }^{a}$ and J NAVARRO-SALAS ${ }^{\mathrm{b}}$ \\ - The Blackett Laboratory, Imperial College London SW7 2BZ, UK \\ b IFIC, Facultad de Fisicas, E-46100 Burjasot (Valencia), Spain
}

Received 3 April 1989

\begin{abstract}
We construct the smallest supergroup $\widetilde{B}(K)$ containing among its generators those of a constraint subgroup $K$, the BRST charge and the corresponding ghost and antighost operators When $\mathrm{K}$ is diff $\mathrm{S}^{1}$ the critical dimension of string theory can be interpreted as a singularity in the carrier space for the irreducible representations of the associated quantum group Some additional physical applicatıons are briefly discussed
\end{abstract}

In this letter we generalize the BRST superalgebra structure given by Bowick and Gursey [1] through the inclusion of ghost operators We also show that many features of BRST theory [2] can be easily understood withın a group approach to quantization (GAQ) $[3,4]$ by puttıng as basic group what we will call the BRST supergroup The prescription for quantizing physical quantities is directly dictated by the group itself and no assumptions are made about Noether invariants The normal ordering arises as a natural consequence of the choice of a particular subalgebra (the polarization subalgebra) [4]

First we introduce the quantum supergroup $\tilde{B}(K)$ which is the smallest supergroup containing an arbitrary bosonic subgroup $\mathrm{K}$ of constraints, the fermionic transformations generated by the BRST charge $\hat{Q}$ and those generated by the ghost and antighost operators $\hat{\Psi}^{* 1}$ and $\hat{\Psi}$, We then describe its treatment withın the GAQ

We begin with an arbitrary Lie algebra of constraints

$$
\left[\hat{K}_{t}, \hat{K}_{\jmath}\right]=f_{l \jmath}{ }^{k} \hat{K}_{k}
$$

For each generator $\hat{K}_{l}$, we introduce a pair of ghost and antıghost operators, $\hat{\Psi}^{* i}$ and $\hat{\Psi}_{\imath}$, which obey the usual anticommutation relations They transform under $\hat{K}_{l}$ according to the adjoint and co-adjoint representations respectively

$$
\left[\hat{K}_{i}, \hat{\Psi}_{\jmath}\right]=f_{l j}{ }^{k} \hat{\Psi}_{k}, \quad\left[\hat{K}_{l}, \hat{\Psi}^{* j}\right]=-f_{l k} \hat{\Psi}^{* k}
$$

We assume the existence of a fermionic generator $\hat{Q}$ satisfying the usual anticommutation relations with ant1ghosts and commutation relations with constraints

$\left\{\hat{Q}, \hat{\Psi}_{l}\right\}=\hat{K}_{l}, \quad\left[\hat{Q}, \hat{K}_{l}\right]=0$

Now the question arıses of which is the smallest superalgebra $\tilde{b}(K)$ containıng $\hat{K}_{l}, \hat{Q}, \hat{\Psi}_{j}, \hat{\Psi}^{* k}$ and 1 and satısfying (1)-(3) The only new generators required to close the superalgebra are $\hat{T}^{\prime} \equiv\left\{\hat{Q}, \hat{\Psi}^{* *}\right\}$, already considered in ref [5] just as a new set of BRS-ınvariant operators The remainıng commutators are

$\left[\hat{Q}, \hat{T}^{\prime}\right]=0,\left[\hat{T}^{\prime}, \hat{T}^{\jmath}\right]=0, \quad\left[\hat{T}^{\prime}, \hat{\Psi}^{* \jmath}\right]=0, \quad\left[\hat{\Psi}_{\jmath}, T_{l}\right]=-f_{j m}{ }^{\prime} \hat{\Psi}^{* m}, \quad\left[\hat{K}_{l}, \hat{T}^{\prime}\right]=-f_{l m}{ }^{\prime} \hat{T}^{m}$

On leave of absence from IFIC, Facultad de Fisıca, E-46100 Burjasot (Valencia), Spain 
Obviously $\tilde{\mathbf{b}}(\mathbf{K})$ is a central extension of the superalgebra $\mathfrak{b}(\mathbf{K})$ generated by $\hat{K}, \hat{Q}, \hat{T}, \hat{\Psi}$, and $\hat{\Psi}^{* k}$, and hence $\widetilde{\mathfrak{B}}(\mathrm{K})$ is a quantum group in the sense of GAQ $[3,4]$

A remarkable property of $\mathfrak{B}(K)$ is that no anomalous term is allowed in (1) as a consequence of (3a) However, it is well known that the subgroup generated by $\hat{K}_{t}, \hat{\Psi}_{\text {, and }} \hat{\Psi}^{* h}$ admits a (negative) anomaly for dim $\mathrm{K}=\infty$ Another interesting property concerns the new generators $\hat{T}_{l}$ Although they do not have any dynamical content, in the sense that no central term appears on the RHS of $\left[\hat{T}_{l}, \hat{T}_{j}\right]$, the subgroup of these new generators affects the definition of the physical vacuum of course $\mathfrak{B}(K)$ can be enlarged so as to contain the set of coordinatemomentum pairs of the physical system under consideration (see below)

Our next task is the construction of a supergroup law for the $U(1)$ centrally extended supergroup $\mathfrak{B}(K)$ in such a way that (1)-(4) correspond to the Lie bracket relations among the right-invariant vector fields $X_{k}^{\mathrm{R}}, X_{q}^{\mathrm{R}}, X_{\psi^{\prime}}^{\mathrm{R}}, X_{\psi_{k}^{*}}^{\mathrm{R}}, X_{T_{m}}^{\mathrm{R}}$ and $X_{b}^{\mathrm{R}}$, where the $\mathrm{U}(1)$ generator now replaces the identity operator $\mathbb{1}$ The group law $1 \mathrm{~S}$

$$
\begin{aligned}
& R^{\prime \prime}{ }_{k}=R^{\prime \prime}{ }_{h} R_{j}^{k}-q^{\prime} f_{m j}{ }^{\prime} R^{\prime \prime}{ }_{k} R^{k}{ }_{r} R^{-1}{ }_{s}^{m} \Psi^{\prime}, \\
& \Psi^{\prime \prime \prime}=\Psi^{\prime \prime}+R^{\prime \prime}{ }_{k} \Psi^{k}-\frac{1}{2} q^{\prime} f_{k s}{ }^{\prime} R^{\prime k}{ }_{m} R^{\prime s}{ }_{n} \Psi^{m} \Psi^{n}, \\
& \Psi_{j}^{* \prime \prime}=\Psi_{j}^{* \prime}+R^{\prime-1 h}{ }_{j} \Psi_{h}^{*}-f_{J s}{ }^{k} R^{\prime-1} m_{k} \Psi^{\prime s} T_{m}+q^{\prime} f_{j s}{ }^{n} R^{\prime-1{ }_{n}} \Psi^{\prime s} \Psi_{k}^{*}, \\
& T_{j}^{\prime \prime}=T_{j}^{\prime}+R^{\prime-1 k}{ }_{j} T_{k}+q^{\prime} R^{\prime-1 k}, \Psi_{k}^{*}, \\
& q^{\prime \prime}=q^{\prime}+q \text {, } \\
& \zeta^{\prime \prime}=\zeta^{\prime} \zeta \exp \left\{1 \frac { 1 } { 2 } \left[\Psi_{l}^{* \prime} R^{\prime \prime}{ }_{j} \Psi^{\prime}-\Psi_{l}^{*} R^{\prime-1}{ }_{j}, \Psi^{\prime \prime}+f_{j k}{ }^{\prime} R^{\prime-1}{ }_{l}^{m} T_{m} \Psi^{\prime j} R^{\prime k}{ }_{s} \Psi^{\varsigma}+\frac{1}{2} q^{\prime} f_{j^{\prime}}{ }^{\prime} \Psi^{\prime} \Psi^{h} \Psi_{l}^{*}\right.\right. \\
& \left.\left.+\frac{1}{2} q^{\prime} f_{j k}{ }^{\prime} R^{\prime}{ }_{l}^{m} \Psi_{m}^{* \prime} \Psi^{\prime} \Psi^{k}+q^{\prime} f_{j k}{ }^{\prime} \Psi^{\prime \prime} R^{\prime}{ }_{m}{ }_{m} \Psi^{m} R^{\prime-1 s}{ }_{l} \Psi_{s}^{*}+\frac{1}{2} q^{\prime} f_{j k}{ }^{\prime} f_{m n}{ }^{\prime} R^{\prime-1}{ }_{1}{ }_{l} R^{\prime}{ }_{,}, \Psi^{\prime \prime} \Psi^{m} \Psi^{n} T_{s}\right]\right\},
\end{aligned}
$$

where $R^{i}$, is the vector realization of the constraint group $\mathrm{K}$ From (5) we immediately derive the left- and right-invariant vector fields They act on the complex functions on $\widetilde{B}(K)$ as linear differential operators Indeed the right-ınvarıant vector fields provide a prequantization or Bohr approxımation to quantization To achieve a true quantization a few additional concepts must be introduced

The Lie algebra cocyle $\Sigma$ defining the central extension $\tilde{b}(K)$ of $b(K)$ (see the first reference in ref [3] for general definitions) can be seen as the restriction to the identity $e$ of an exact left-invariant two-form $\mathrm{d} \Theta\left(\left.\mathrm{d} \Theta\right|_{\imath}=\Sigma\right)$ The (quantization) 1-form $\Theta$ is the $\mathrm{U}(1)$ component $\left(\vartheta^{\mathrm{L}(\zeta)} \equiv \Theta\right)$ of the left-invariant canonical one-form $\vartheta^{\mathrm{L}}$ The kernel of $\Sigma$ can now be realızed as a subalgebra $g_{\Theta}$ of left-ınvariant vector fields on $\widehat{\mathfrak{B}}(\mathrm{K})$ annihilating $\mathrm{d} \Theta$ as well as $\Theta$, called the characteristic subalgebra From the physical point of view the generators in $g_{\Theta}$ are those which are not canonically conjugate to any other in the whole group $\mathfrak{B}(K)$ The quantization form $\Theta$ of $\tilde{B}(K)$ is easily calculated (using duality on $\tilde{X}^{\mathrm{L}}, \Theta\left(\tilde{X}_{\zeta}^{\mathrm{L}}\right)=1, \Theta\left(\tilde{X}_{g \neq \zeta}^{\mathrm{L}}\right)=0$ )

$\Theta=\frac{1}{2}\left(\Psi_{l}^{*} \mathrm{~d} \Psi^{l}+\Psi^{\prime} \mathrm{d} \Psi_{l}^{*}\right)-\frac{1}{2} f_{m n}^{\prime} \Psi^{m} \Psi^{n} \mathrm{~d} T_{l}+\frac{\mathrm{d} \zeta}{1 \zeta}$,

and one finds that $\mathrm{g}_{\Theta}$ is generated by $X_{q}^{\mathrm{L}}, X_{h^{\prime}}^{\mathrm{L}}, X_{T}^{\mathrm{L}}, l, J=1, \quad \operatorname{dim} \mathrm{K}$ From $\Theta$ we can directly obtain the Noether invariants, defined in our approach as $\Theta\left(X_{a}^{\mathrm{R}}\right), \forall X_{a}^{\mathrm{R}} \in \mathfrak{b}(\tilde{\mathrm{K}})$, which, however, are not needed in the quantum theory

$$
\begin{aligned}
& \Theta\left(\tilde{X}_{(q)}^{\mathrm{R}}\right)=-\frac{1}{2} f_{m n}{ }^{\prime} \Psi^{m} \Psi^{n} \Psi_{t}^{*} \equiv \mathfrak{Q}, \\
& \Theta\left(\tilde{X}_{\left(h^{\prime}\right)}^{\mathrm{R}}\right)=-f_{l m}{ }^{s}\left(\Psi^{m} \Psi_{s}^{*}-f_{s n}{ }^{k} T_{k} \Psi^{m} \Psi^{n}\right) \equiv \mathfrak{J}_{1}, \\
& \Theta\left(\tilde{X}_{\left(\Psi^{\prime}\right)}^{\mathrm{R}}\right)=\left(\Psi_{l}^{*}+f_{t h}{ }^{m} \Psi^{k} T_{m}\right) \equiv \mathfrak{f}_{l}, \\
& \Theta\left(\tilde{X}_{\left(\Psi^{*}\right)}^{\mathrm{R}}\right)=\Psi^{\prime} \equiv \mathfrak{f}^{* l}, \\
& \Theta\left(\tilde{X}_{\left(l_{l}\right)}^{\mathrm{R}}\right)=-\frac{1}{2} f_{m h}{ }^{\prime} \Psi^{m} \Psi^{h} \equiv \mathfrak{I}^{\prime}
\end{aligned}
$$


The only independent invariants are $\Theta\left(X_{\psi_{i}}^{\mathrm{R}}\right) \equiv \mathrm{f}_{l}$ and $\Theta\left(X_{\Psi_{j}^{*}}^{\mathrm{R}}\right) \equiv \mathfrak{f}^{* \prime}$ The classical BRST charge, $\mathbb{Q} \equiv \Theta\left(X_{q}^{\mathrm{R}}\right)$, has the standard form

$\mathbb{Q}=\mathfrak{f}^{* i} \mathfrak{J}_{t}+\frac{1}{2} f_{m n} \mathfrak{f}_{i} \mathfrak{f}^{* m \mathfrak{F}} \mathfrak{f}^{* n}$,

where the plus sign appears because $\mathfrak{I}_{l} \equiv \Theta\left(X_{k^{\prime}}^{\mathrm{R}}\right)$ is the total "angular momentum"

Another subalgebra required in the quantization process to reduce the representation is a subalgebra of leftinvariant vector fields which impose restrictions on the complex functions on $\widetilde{B}(K)$ We generalize in this way the restriction which takes place in ordinary quantum mechanics when going from wave functions $\Theta(x, p)$ to $\Phi(\boldsymbol{x}), \Phi(\boldsymbol{p})$ or $\Phi(\boldsymbol{x}+\boldsymbol{p})$ A full polarization (respectively polarızation) $\mathfrak{P}$ is a maximal left-subalgebra containing $g_{\theta}$ (respectively a proper subalgebra of $g_{\theta}$ ) and excluding the central generator [4,6] (to prevent pairs of canonically conjugate variables occurring in $\mathfrak{P}$ ) Note that in some special (but important) cases related to anomalies there is an obstruction to the full polarization subalgebra For instance, in string theory a full polar1zation should be made out of the Virasoro generators as well as half the loop space generators $\alpha_{n}^{\mu}$, but it does not close $[7,6]$

Finally, the quantum operators are the right-invariant vector fields acting on wave functions, $1 \mathrm{e}$, those complex functions on $\widetilde{\mathfrak{B}}(\mathbf{K})$ which satisfy

$X_{\zeta}^{\mathrm{R}} \psi \equiv 1 \zeta(\partial / \partial \zeta) \psi=1 \psi, \quad \mathrm{X}^{\mathrm{L}} \psi=0 \quad \forall X^{\mathrm{L}} \in \mathfrak{P}$

Irrespective of the dımension of $\mathfrak{B}(K)$ (finite or infinite) there always exists a full polarization (called natural polarization [8]) containing the $\Psi_{j}^{*}, \forall j=1$, dim K

$\mathfrak{P}^{\mathrm{N}}=\left\langle X_{q}^{\mathrm{L}}, X_{k^{\prime}}^{\mathrm{L}}, X_{\Psi_{j}^{*}}^{\mathrm{L}}, X_{T_{k}}^{\mathrm{L}}\right\rangle$

Solving eq $(8)$ for the full polarization $\mathfrak{P}^{\mathrm{N}}$ leads to the Fock states

$|1\rangle \sim \zeta W, \quad\left|\Psi^{\prime}\right\rangle \sim \zeta W \Psi^{\prime}, \quad, \quad\left|\Psi^{\prime 1} \quad \Psi^{\prime n}\right\rangle \sim \zeta W \Psi^{i 1} \quad \Psi^{\prime n}$,

where $l_{h}=1, \quad, \operatorname{dim~K}$ and the weight function $W$ is given by

$W=\exp \left[1 \frac{1}{2}\left(\Psi_{n}^{*} \Psi^{n}-f_{i j}{ }^{k} \Psi^{\prime} \Psi^{\prime} T_{k}\right)\right]$

The action of the operators $X^{\mathrm{R}}$ on the basic states $|1\rangle,\left|\Psi^{t}\right\rangle$ is

$X_{q}^{\mathrm{R}}|1\rangle=0, \quad X_{q}^{\mathrm{R}}\left|\Psi^{\prime}\right\rangle=-\frac{1}{2} f_{j^{\prime}}{ }^{\prime}\left|\Psi^{\prime} \Psi^{k}\right\rangle, \quad X_{k^{\prime}}^{\mathrm{R}}|1\rangle=0, \quad X_{k^{\prime}}^{\mathrm{R}}\left|\Psi^{\prime}\right\rangle=-f_{m^{\prime}}{ }^{\prime}\left|\Psi^{m}\right\rangle, \quad X_{\psi^{\prime}}^{\mathrm{R}}|1\rangle=0$,

$X_{\psi^{\prime}}^{\mathrm{R}}\left|\Psi^{\prime}\right\rangle=\delta_{j}^{\prime}|1\rangle, \quad X_{\Psi_{j}^{*}}^{\mathrm{R}}|1\rangle=1\left|\Psi^{\prime}\right\rangle, \quad X_{\Psi_{j}^{*}}^{\mathrm{R}}\left|\Psi^{\prime}\right\rangle=1\left|\Psi^{\prime} \Psi^{\prime}\right\rangle, \quad X_{T_{1}}^{\mathrm{R}}|1\rangle=-1 \frac{1}{2} f_{m n}\left|\Psi^{m} \Psi^{n}\right\rangle$,

$X_{l}^{\mathrm{R}}\left|\Psi^{\prime}\right\rangle=-1 \frac{1}{2} f_{m n}{ }^{\prime}\left|\Psi^{\prime} \Psi^{m} \Psi^{n}\right\rangle$

Their action on arbitrary states $\left|\Psi^{i 1} \Psi_{n}^{n}\right\rangle$ follows from the factorization property of wave functions From (12) we can find relations among operators, e $\mathrm{g}$

${ }_{1} \boldsymbol{X}_{q}^{\mathrm{R}}=\boldsymbol{X}_{k^{\prime}}^{\mathrm{R}} \boldsymbol{X}_{\Psi_{i}^{*}}^{\mathrm{R}}+\frac{1}{2} f_{m n}{ }^{i} \boldsymbol{X}_{\psi_{i}}^{\mathrm{R}} \boldsymbol{X}_{\Psi_{m}^{*}}^{\mathrm{R}} \boldsymbol{X}_{\Psi_{n}^{*}}^{\mathrm{R}}$.

The group $\mathfrak{B}(K)$ is special since, unlıke for more other groups, the weight function $|1\rangle$ cannot be considered as the physical vacuum from which the creation operators generate the Fock space The state $|1\rangle$ is not annihilated by the right-invariant vector fields associated with the algebra $g_{\theta}$ (of "non-step" operators) as is normally required, since $X_{T}^{\mathrm{R}}|1\rangle \neq 0$ Thus we must choose an appropriate vacuum $|0\rangle$ from the polarized wave functions (satisfyıng $X_{a}^{\mathrm{R}}|0\rangle=0$ if the corresponding $X_{a}^{\mathrm{L}}$ lies in $\mathrm{g}_{\Theta}$ ) When the constraint group $\mathrm{K}$ is finite-dimensional we find the "Dirac sea", $|0\rangle \equiv \zeta W \prod_{i} \Psi^{i}, l=1$, , dim K

The situation is much more complicated when $\mathrm{K}$ is infinite-dimensional and a non full polarization can be chosen (a fact which is related to an anomalous term in the group $\mathrm{K}$ ) We shall be concerned from now on with the case $\mathrm{K}=\operatorname{diff} \mathrm{S}^{1}$ for which the matrix $R_{m}^{k}$ of the general group law (5) is 
$R_{m}^{k}=\delta_{m}^{h}+1(2 m-k) 1^{k-m}+\frac{(1)^{2}}{2^{\prime}} \sum_{m_{1}+n_{2}+m=h}\left[m^{2}-m\left(n_{1}+n_{2}\right)+2 n_{1} n_{2}\right] l^{m_{1}} l^{m_{2}}+$,

which can be derived from the exact Virasoro group law given in ref [6] The choice of polarization and vacuum analogous to the finite-dimensional one described above leads to the infinite wedge product representations (or Just the semi-infinite ones [9] if the condition $X_{T_{A}}^{\mathrm{R}}|0\rangle=0$ is imposed for only $k \geqslant 0$ ) However, here we are interested in a more physical quantization of $\mathfrak{B}\left(\operatorname{diff}^{\prime} S^{1}\right.$, which corresponds in this approach to a different type of polarization which is absent from the finite-dimensional case

As already mentioned, no anomalous term is allowed in (1), (3a) Thus, for $K=\operatorname{diff~}^{1}$ the conformal anomaly $c$ in the commutator

$$
\left[L_{n}, L_{m}\right]=(n-m) L_{n+m}+\frac{1}{2}\left(c n^{3}-c^{\prime} n\right) \delta_{n-m}
$$

must vanısh On the other hand there are no restrictions on $c^{\prime}$, which parametrizes a family of pseudo-extensions (trivial extensions behaving under the GAQ like true extensions) $[3,6]$ The commutators for $\mathfrak{B}\left(\operatorname{diff}^{1}\right)$ are (2), (3b), (4) with $f_{m n}{ }^{\prime}=(m-n) \delta_{m+n}^{\prime},(15)$ with $c=0$, and $\left\{Q, \Psi_{n}\right\}=L_{n}-\frac{1}{24} c^{\prime} \delta_{n 0}$ For $c^{\prime} \neq 0$ the character1stic subalgebra is

$\mathrm{g}_{\Theta}=\left\langle X_{\omega}^{\mathrm{L}}, X_{T_{n}}^{\mathrm{L}}\right\rangle$,

and our supergroup $\mathfrak{B}\left(\operatorname{diff} S^{1}\right)$ admits a new class of polarızations of standard type [8] which are not full polarizations (they cannot contain all the generators $X_{I_{n}}^{\mathrm{L}}, n \in \mathbb{Z}$ ) Out of those only the following two are not equivalent

$\mathfrak{P}^{\mathrm{S}}=\left\langle X_{\eta^{n} \leqslant 0}^{\mathrm{L}}, X_{\Psi^{n}<0}^{\mathrm{L}}, X_{\Psi_{n>0}^{*}}^{\mathrm{L}}, X_{T_{n \geqslant 0}}^{\mathrm{L}}, X_{\Psi_{0}^{*}}^{\mathrm{L}}, X_{q}^{\mathrm{L}}\right\rangle, \quad \mathfrak{P}^{\prime \mathrm{S}}=\left\langle X_{I^{n} \leqslant 0}^{\mathrm{L}}, X_{\psi^{n}<0}^{\mathrm{L}}, X_{\Psi_{n}^{*}>0}^{\mathrm{L}}, X_{I_{n} \geqslant 0}^{\mathrm{L}}, X_{\Psi_{0}}^{\mathrm{L}}\right\rangle$

Following the general strategy of takıng polarizations leading to maximal reduction, we choose (17a) (in the usual terminology these two choices correspond to vacua with ghost numbers $-\frac{1}{2}$ and $+\frac{1}{2}$ respectively) From $(8 \mathrm{a}),(8 \mathrm{~b})$ the polarized wave functions can be calculated in the parametrization for the Virasoro group given in ref [6] The explicit calculations will be presented elsewhere Here we only remark that since (17a) is a polarization of standard type (1t contains both particle and anti-particle operators) the vacuum is essentially a weight function $|0\rangle \sim \zeta W$ The state $|0\rangle$ is annihilated by $X_{i}^{\mathrm{R}}$ and also by the operators $X_{\mu^{n}>0}^{\mathrm{R}}, X_{\psi^{n}>0}^{\mathrm{R}}, X_{\Psi_{n<0}^{*}}^{\mathrm{R}}, X_{T_{n<0}}^{\mathrm{R}}, X_{\psi^{0}}^{\mathrm{R}}$, which play the role of annihilation operators

So far we have considered only the subgroup $\mathfrak{B}(K)$ associated with the constrant group $K$, and not the ord1nary coordinate and momentum operators We will now show how the particular subgroup $\tilde{\mathfrak{B}}$ (diff $S^{1}$ ) can be enlarged so as to contain the phase space operators $\alpha_{n}^{\mu}$ for open strings [7] (the loop space group $\mathbb{P R}^{d-11}$ ) The coordinate-momentum pairs are the $\left(\alpha_{n}, \alpha_{-n}\right)$, and the bigger supergroup $\mathfrak{B}$ (diff $S^{1}(S) \mathfrak{R} \mathbb{R}^{1 d-1}$ ) has the add1tional non-zero commutators

$$
\begin{aligned}
& {\left[\hat{\alpha}_{n}^{\mu}, \hat{\alpha}_{m}^{\nu}\right]=n g^{\mu \nu} \delta_{n-m} \hat{\mathbb{1}} \equiv \sum_{n m}^{\mu \nu} \hat{1}, \quad\left[\hat{L}_{n}, \hat{\alpha}_{m}^{\mu}\right]=m \hat{\alpha}_{n+m}^{\mu} \equiv F_{n m}{ }^{k} \hat{\alpha}_{k}^{\mu}, \quad\left[\hat{Q}, \hat{\alpha}_{n}^{\mu}\right]=\hat{\pi}_{n}^{\mu},} \\
& {\left[\hat{L}_{n}, \hat{\pi}_{m}^{\mu}\right]=m \hat{\pi}_{n+m}^{\mu} \equiv F_{n m}{ }^{k} \hat{\pi}_{k}^{\mu}, \quad\left[\hat{\pi}_{n}^{\mu}, \hat{\alpha}_{m}^{\nu}\right]=-n m g^{\mu \nu} \hat{\Psi}^{* n+m} \equiv-\sum_{n h}^{\mu \nu} F_{m}{ }^{h} \hat{\Psi}^{* \mathrm{~s}},} \\
& \left\{\hat{\pi}_{n}^{\mu}, \hat{\pi}_{m}^{\nu}\right\}=n m g^{\mu \nu} \hat{T}^{n+m} \equiv \sum_{n h}^{\mu \nu} F_{m s}{ }^{k} \hat{T}^{s}, \quad\left\{\hat{\pi}_{n}^{\mu}, \hat{\Psi}_{m}\right\}=m \hat{\alpha}_{n+m}^{\mu} \equiv F_{n m}{ }^{k} \hat{\alpha}_{k}^{\mu},
\end{aligned}
$$

where again we had to introduce a new set of (fermionic) operators $\hat{\pi}_{n}^{\mu}$ to close the algebra The anticommutators among these new fermionic variables do not admit a central term so that no dynamical role can be attributed to them Applying the GAQ to $\tilde{B}\left(\operatorname{diff}^{1}(s) \mathbb{R}^{1 d-1}\right.$ ) exhibits in a natural way the critical dimension of string theory, but clearly the argument will be different from the standard one [10] since in our approach the condition $Q^{2}=0$ is fulfilled trivially Indeed $c$ also vanishes in $\mathfrak{B}$ (diff $S^{1}(s) \mathcal{L R}^{1 d-1}$ ), and the usual mechanism for anomaly cancellation does not exist However, the physical anomaly shows up when writing down the operators $\left(\tilde{X}^{\mathrm{R}}\right)$, which turn out to be functions of more than the basic operators, $1 \mathrm{e}$, those involved in the commutators produc- 
ing non-trivial central terms Only for critical values of the constant parameters characterizing the whole algebra all operators $\tilde{X}^{\mathrm{R}}$ can be written in terms of the basic operators (in our present case $\hat{\alpha}$ 's, $\hat{\Psi} *$ 's and $\hat{\Psi}$ 's, see later)

The only (non full) polarization leading to unitary (and physical) representations is the following one

$\mathfrak{P}^{\mathrm{S}}=\left\langle\tilde{X}_{(q)}^{\mathrm{L}}, \tilde{X}_{(l n \leqslant 0)}^{\mathrm{L}}, \tilde{X}_{(T n>0)}^{\mathrm{L}}, \tilde{X}_{\left(\Psi_{n>0)}^{*}\right.}^{\mathrm{L}}, \tilde{X}_{\left(\Psi_{n<0)}\right.}^{\mathrm{L}}, \tilde{X}_{\left(\alpha_{n<0)}\right.}^{\mathrm{L}}, \tilde{X}_{(\pi n<0)}^{\mathrm{L}}\right\rangle$

(19) is of standard type and contains (17a) The integration of the polarization equations $\tilde{X}^{\mathrm{L}} \Psi=0 \forall \tilde{X}^{\mathrm{L}} \in \mathfrak{P}^{\mathrm{S}}$ will be given to the third order in the group parameters (Of course, the main results do appear at this level) The wave functions are

$$
\begin{aligned}
& |0\rangle \sim \zeta W_{1}(q, 1, \Psi) W_{2}\left(\Psi, \Psi^{*}, T, \alpha, \pi, 1\right) \equiv \zeta W, \\
& \left|l^{l>0}\right\rangle \sim \zeta W\left(l-\sum_{k>0} k k^{k-k}-q \Psi^{\prime}+\right) \\
& \left|T_{l<0}\right\rangle \sim \zeta W\left(T_{l}-\sum_{k<0} f_{m l}{ }^{k} l^{m} T_{k}+\frac{1}{2} \sum_{k \leqslant 0} \sum_{s k}^{\mu \nu} F_{t m}{ }^{s} \pi_{\mu}^{k} \pi_{\nu}^{m}-q \Psi_{l}^{*}+\right), \\
& \left|\pi_{\mu}^{n>0}\right\rangle \sim \zeta W\left(\pi_{\mu}^{n}-\sum_{k>0} F_{m k}{ }^{n} l^{m} \pi_{\mu}^{k}+q \alpha_{\mu}^{n}+\right) \\
& \left|\alpha_{\mu}^{n>0}\right\rangle \sim \zeta W\left(\alpha_{\mu}^{n}-\sum_{k>0} F_{m k}{ }^{n} l^{m} \alpha_{\mu}^{k}-\sum_{m<0} F_{m k}{ }^{n} \Psi^{m} \pi_{\mu}^{k}+\right) \\
& \left|\Psi^{t>0}\right\rangle \sim \zeta W\left(\Psi^{i}+\sum_{k \geqslant 0} f_{m k} l^{m} \Psi^{k}+\right), \\
& \left|\Psi_{i<0}^{*}\right\rangle \sim \zeta W\left(\Psi_{\imath}^{*}-\sum_{k<0} f_{m t}{ }^{k} l^{m} \Psi_{k}^{*}-\sum_{k<0} f_{m t}{ }^{k} T_{k} \Psi^{m}-\frac{1}{2} \sum_{k>0} \sum_{s k}^{\mu \nu} F_{t m}{ }^{s} \pi_{\mu}^{k} \alpha_{\nu}^{m}+\frac{1}{2} \sum_{k>0} \sum_{s k}^{\mu \nu} F_{l m}{ }^{s} \alpha_{\mu}^{k} \pi_{\nu}^{m}+\right),
\end{aligned}
$$

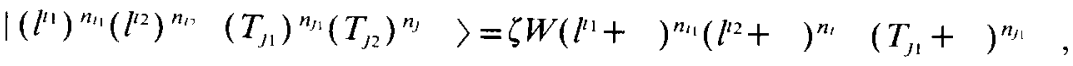

where the factors $W_{1}$ and $W_{2}$ of the weight function associated with the vacuum are

$$
\begin{aligned}
& W_{2}=1-\frac{1}{2} 1\left(\sum_{n \geqslant 0} \Psi_{n}^{*} \Psi^{n}-\sum_{n<0} \Psi_{n}^{*} \Psi^{n}-\sum_{n m k<0} f_{n m}{ }^{k} \Psi^{n} \Psi^{m} T_{k}+2 \sum_{\substack{n>0 \\
m<<<}} f_{n k}{ }^{m} \Psi_{m}^{*} \Psi^{n} l^{k}+\right) \\
& -\frac{1}{2} 1\left(\sum_{n \geqslant 0} \sum_{n k}^{\mu \nu} \alpha_{\mu}^{n} \alpha_{\nu}^{k}-\sum_{\substack{n \geqslant 0 \\
m k<0}} F_{k m} \sum_{n j}^{\mu \nu} \alpha_{\mu}^{n} \pi_{\nu}^{m} \Psi^{k}-\sum_{\substack{n \leqslant 0 \\
n m>0}} F_{k m} \sum_{n j}^{\mu \nu} \alpha_{\mu}^{n} \alpha_{\nu}^{m} l^{k}+\right), \\
& W_{1}=\tilde{W}_{1}(l)-\frac{1}{24} c^{\prime} q \sum_{n \geqslant 0} \Psi^{n}\left[n\left(-1^{-n}+2 \sum_{m>0} m l^{m} l^{-n-m}-3 \sum_{m k>0} m k l^{m} l^{k} l^{-n-m-h}+\right)\right. \\
& \left.+\sum_{m \geqslant 0}(-m-n) l^{-m-n}\left(-m l^{m}+\sum_{\substack{j, s>0 \\
j+s=m}} j s l^{s}-\sum_{\substack{j s r>0 \\
j+s+r=m}} j s r l l^{s} l^{r}+\right)\right] \\
& \tilde{W}_{1}(l)=1+\frac{1}{24} \mathcal{c}^{\prime}\left(\sum_{n>0}-n l^{n} l^{-n}+\sum_{n, m>0} n m l^{n} l^{m} l^{-n-m}-\sum_{n m k>0} n m k l^{n} l^{m} l^{k} l^{-n-m-k}+\right)
\end{aligned}
$$


$\tilde{W}_{1}(l)$ is just the weight function of the Virasoro subgroup itself and $W_{1}(l, q, \Psi)$ is the weight function which would correspond to the Bowick-Gursey subalgebra [1] (here generated by $\widetilde{X}_{l^{n}}, \widetilde{X}_{\psi^{m}}, \widetilde{X}_{q}$ )

The physical operators, the right-invariant vector fields, are well-defined differential operators acting on (20) We will not write the explicit action of all of them, limiting ourselves to those calculations that are strictly necessary for determining the critical values of $c^{\prime}$ and $d$ (the spacetime dimension) The action of

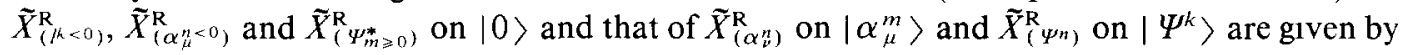

$$
\begin{aligned}
& \tilde{X}_{(l-n<0)}^{\mathrm{R}}|0\rangle=-\frac{1}{2} c^{\prime} n\left|l^{n}\right\rangle+\frac{1}{2} 1 F_{-n m}^{\prime} \sum_{r s}^{\mu \nu}\left|\alpha_{\mu}^{r} \alpha_{\nu}^{m}\right\rangle-1 f_{r-n}^{m}\left|\Psi_{m}^{*} \Psi^{r}\right\rangle+ \\
& =-\frac{1}{12} c^{\prime} n\left|l^{n}\right\rangle+\frac{1}{2} 1 g^{\mu \nu} \sum_{0<m<n} m(n-m)\left|\alpha_{\mu}^{n-m} \alpha_{\nu}^{m}\right\rangle-1 \sum_{0 \leqslant r<n}(r+n)\left|\Psi_{r-n}^{*} \Psi^{r}\right\rangle+, \\
& \tilde{X}_{(\alpha m<0)}^{\mathrm{R}}|0\rangle=-1 \Sigma_{n m}^{\mu \nu}\left|\alpha_{\nu}^{n}\right\rangle+, \quad \tilde{X}_{\left(\Psi_{m \geqslant 0}^{*}\right)}^{\mathrm{R}}|0\rangle=-1\left|\Psi^{m}\right\rangle+, \\
& \widetilde{X}_{\left(\alpha_{v}<0\right)}^{\mathrm{R}}\left|\alpha_{\mu}^{n}\right\rangle=-1 \sum_{p r}^{\nu \rho}\left|\alpha_{p}^{P} \alpha_{\mu}^{n}\right\rangle+r \delta_{\mu}^{\nu}\left|l^{n-r}\right\rangle+\quad, \quad \tilde{X}_{\left({ }^{2}<0\right)}^{\mathrm{R}}\left|\Psi^{p}\right\rangle=-1\left|\Psi_{r}^{*} \Psi^{P}\right\rangle+(2 r-p)\left|l^{p-r}\right\rangle+
\end{aligned}
$$

The representations obtained in this way are in general reducible The reduction can then be achieved by choosing a vacuum (the weight function in the present case) from which the non-trivial action of the operators generate the irreducible carrier space It must be stressed that this construction is rather different from the ordinary abstract one of the Lie algebra representation There, all the states are obtained from the vacuum through the action of the creation operators which are, by definition, linearly independent On the contrary, our states, in particular the vacuum, are explicit functions on the (super)group whose linear dependence or independence is prior to the action of the operators The null vectors appear as truly null linear combination of vector states and then the carrier space

$$
\prod\left(\tilde{X}_{\left(l^{n<0}\right)}^{\mathrm{R}}\right)^{p_{n}}\left(\tilde{X}_{\left(\alpha \alpha_{i}^{(<0}\right)}^{\mathrm{R}}\right)^{t_{s}}\left(\tilde{X}_{\left(\psi^{\prime m<0}\right)}^{\mathrm{R}}\right)^{q_{m}}\left(\tilde{X}_{\left(\Psi_{k \geqslant 0}^{*}\right)}^{\mathrm{R}}\right)^{r_{k}}|0\rangle
$$

is irreducible From (22) we deduce that the structure of the state $\tilde{X}_{(1-k<0)}^{\mathrm{R}}|0\rangle$ is identical with that of the state

$$
\left(-\frac{1}{1} \sum_{m, r} f_{m-k} r \tilde{X}_{\left(\psi^{r}\right)}^{\mathrm{R}} \tilde{X}_{\left(\psi_{m}^{*}\right)}^{\mathrm{R}}+\frac{1}{21} g_{\mu \nu} \tilde{X}_{\left(\alpha_{\bar{\nu}} \bar{p}^{k-m}\right)}^{\mathrm{R}} \tilde{X}_{\left(\alpha_{\mu}^{m}\right)}^{\mathrm{R}}\right)|0\rangle,
$$

although only for special values of $d$ and $c^{\prime}$ they are proportional

$$
d=26, \quad c^{\prime}=24
$$

In fact, the lowest orders of (24) are

$$
\begin{aligned}
& -1 \sum_{0 \leqslant m<h}(m+k)\left|\Psi_{m-h}^{*} \Psi^{m}\right\rangle+\frac{1}{2} 1 g^{\mu \nu} \sum_{0>m>-h}(-m)(k+m)\left|\alpha_{\mu}^{h+m} \alpha_{\nu}^{-m}\right\rangle \\
& +\sum_{0 \leqslant m<h}(m+k)(m-2 k)\left|l^{h}\right\rangle-\frac{1}{2} g_{\mu \nu} \sum_{0>m>-h}(-m) g^{\mu \nu}(-k-m)\left|l^{h}\right\rangle \\
& =-\left[\left(\frac{13}{6}-\frac{1}{12} d\right) k^{3}-\left(\frac{1}{6}-\frac{1}{12} d\right) k\right]\left|l^{h}\right\rangle-1 \sum_{0 \leqslant m<h}(m+k)\left|\Psi_{m-h}^{*} \Psi^{m}\right\rangle \\
& +\frac{1}{2} 1 g^{\mu \nu} \sum_{0>m>-h}(-m)(k+m)\left|\alpha_{\mu}^{h+m} \alpha_{\nu}^{-m}\right\rangle
\end{aligned}
$$

For these values the irreducible carrier space is made out of states created by just the operators $\tilde{X}_{(\Psi)}^{\mathrm{R}}, \tilde{X}_{\left(\psi^{*}\right)}^{\mathrm{R}}$ and $\tilde{X}_{(\alpha)}^{\mathrm{R}}$ from the vacuum (similarly, the operators $\tilde{X}_{(\pi)}^{\mathrm{R}}, \tilde{X}_{(T)}^{\mathrm{R}}$ and $\tilde{X}_{(q)}^{\mathrm{R}}$ also admit a Sugawara-like construction) The critical values thus appear as a singularity of the carrier space for the irreducible representations of the corresponding supergroup $\mathfrak{B}$ (diff $S^{1}, \operatorname{diff}^{1}(S) R^{1 d-1}$ ) In a more general situation in which the Minkowski space is replaced with a finite-dimensional semisimple group $\mathrm{G}$, the Kac-Moody group $\widetilde{\Omega} \mathrm{G}$ substitutes $\mathfrak{Q R}^{1 d-1}$ Now, the same mechanısm which has led to the Sugawara-like construction of $\widetilde{X}^{\mathrm{R}}$ also produces, for level one 
(as a critical value), the vertex operator construction of the generators outside the torus of $G$ ( 1 e the step operators ) [11]

Let us go back to the general case of an arbitrary constraint group $K$ The supergroup $\widetilde{\mathcal{B}}(\mathrm{K})$ generalizes the customary $U(1)$ phase invariance of wave functions [4] and geometrically this means dealing with a $\mathfrak{B}(K)$ principal bundle instead of the usual $U(1)$ bundle of the unconstrained dynamical systems The infinitesimal version of the $U(1)$ phase invariance, (8a), is now extended to

$\tilde{X}^{\mathrm{R}} \Psi=d D(X) \Psi, \quad \forall X \in \tilde{\mathrm{b}}(\mathrm{K})$,

where $d D$ is a linear representation of $\tilde{\mathrm{b}}(\mathrm{K})$ [4] which eventually may be one-dimensional Thus, for instance, when $\mathrm{K}$ is the $\mathrm{SO}$ (3) subgroup of the Galıle group, (8b) and (26) provide the wave functions corresponding to a free particle moving with fixed angular momentum (given by the index $f$ of an irreducible representation $d D^{(1)}$ of $\mathrm{SO}(3)$ in (26)) For $\mathrm{K}=\operatorname{diff} \mathrm{S}^{1}$ the general scheme reproduces, for the critical points already obtained, the $Q$-cohomology (with ghost number $-\frac{1}{2}$ ) of physical states in string theory as well as the ordinary Virasoro constraints $\hat{L}_{n \geqslant 0} \Psi=\delta_{n 0} \Psi$ It is realized by the following right-invariant differential conditions [11]

$X_{q}^{\mathrm{R}} \Psi=X_{\Psi^{n} \geq 0}^{\mathrm{R}} \Psi=X_{\Psi_{n<0}^{*}}^{\mathrm{R}} \Psi=X_{T_{n \leqslant 0}}^{\mathrm{R}} \Psi=0, \quad X_{n=0}^{\mathrm{R}} \Psi=\delta_{n 0} \Psi$

As a final comment we want to remark that the present mechanısm can also be applied to (non-abelian) gauge theory In that case the differential equations (26) implement the Gupta-Bleuler conditions and also the zero ghost number of the physical states Much work is being done in this direction

V A thanks C J Isham for many discussions and for his hospitality at Imperial College R L wishes to thank the Dr Carl Dussberg-Stıftung for financial support J N -S thanks J W Valle for useful discussions

\section{References}

[1] M J Bowick and F Gursey, Phys Lett B 175 (1986) 182, Nucl Phys B 283 (1987) 331

[2] L A Batalin and G A Vilkovisky, Phys Lett B 69 (1977) 309 ,

D McMullan, J Math Phys 28 (1987) 428,

R Loll, Commun Math Phys 119 (1988) 509

[3] V Aldaya and J A de Azcarraga, J Math Phys 23 (1982) 1297, Phys Lett B121 (1983) 331, Ann Phys (NY) 165 (1985) 484

[4] V Aldaya, J Navarro-Salas and A Ramirez, Commun Math Phys 112 (1989) 541

[5] T Kawal, Phys Lett B 168 (1986) 355

[6] V Aldaya and J Navarro-Salas, preprint Imperial/TP/87-88/26

[7] M J Bow1ck and S G Rajeev, Phys Rev Lett 58 (1987) 58

[8] H P Jacobsen and V Kac, in Lecture Notes in Physıcs, Vol 226, ed N Sanchez (Springer, Berlın, 1985) pp 1-20, V Aldaya and J Navarro-Salas, Commun Math Phys 113 (1987) 375

[9] I B Frenkel, H Garland and G J Zuckerman, Proc Natl Acad Scı (USA) 83 (1986) 8442

[10] S Hwang, Phys Rev D 28 (1983) 2614

[11] V Aldaya, $\mathbf{R}$ Loll and $\mathbf{J}$ Navarro-Salas, in preparation 